\title{
Tracing Catalytic Conversion on Single Zeolite Crystals in 3D with Nonlinear Spectromicroscopy
}

\author{
Katrin F. Domke, ${ }^{*}{ }^{\dagger}$ T. Alexander Riemer, ${ }^{\dagger}$ Gianluca Rago, ${ }^{\dagger}$ Andrei N. Parvulescu, ${ }^{\dagger}$ \\ Pieter C. A. Bruijnincx, ${ }^{\ddagger}$ Annika Enejder, ${ }^{\mathscr{T}}$ Bert M. Weckhuysen, ${ }^{\dagger}$ and Mischa Bonn ${ }^{\dagger, \S}$ \\ ${ }^{\dagger}$ FOM Institute AMOLF, Science Park 104, NL-1098 XG Amsterdam, The Netherlands \\ ${ }^{\ddagger}$ Inorganic Chemistry and Catalysis Group, Utrecht University, Universiteitsweg 99, NL-3584 CG Utrecht, The Netherlands

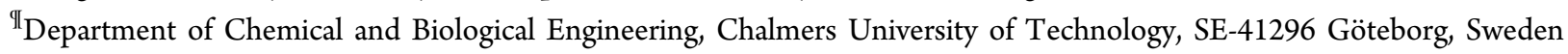 \\ ${ }^{\S}$ Max Planck Institute for Polymer Research, Ackermannweg 10, D-55128 Mainz, Germany
}

\section{Supporting Information}

\begin{abstract}
The cost- and material-efficient development of nextgeneration catalysts would benefit greatly from a molecular-level understanding of the interaction between reagents and catalysts in chemical conversion processes. Here, we trace the conversion of alkene and glycol in single zeolite catalyst particles with unprecedented chemical and spatial resolution. Combined nonlinear Raman and two-photon fluorescence spectromicroscopies reveal that alkene activation constitutes the first reaction step toward glycol etherification and allow us to determine the activation enthalpy of the resulting carbocation formation. Considerable inhomogeneities in local reactivity are observed for micrometer-sized catalyst particles. Product ether yields observed on the catalyst are ca. 5 times higher than those determined off-line. Our findings are relevant for other heterogeneous catalytic processes and demonstrate the immense potential of novel nonlinear spectromicroscopies for catalysis research.
\end{abstract}

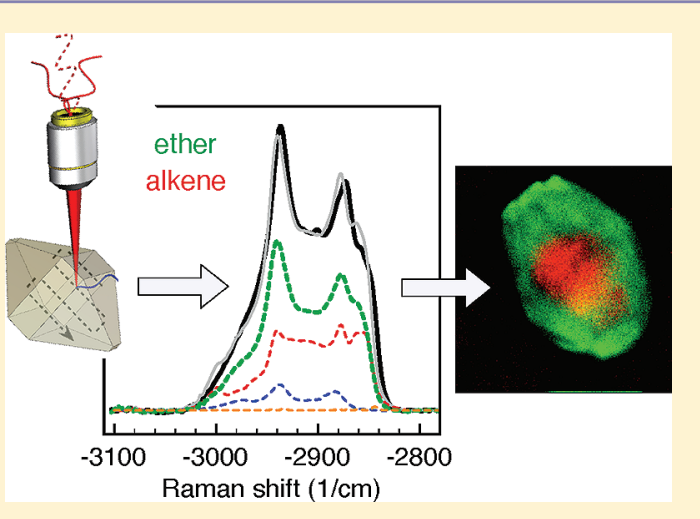

\section{INTRODUCTION}

Heterogeneous catalysis lies at the basis of industrial production of chemicals and energy carriers. Although the commonly employed trial-and-error approach to catalyst optimization has led to well-established reaction protocols for specific chemical conversions, such efforts are generally cumbersome as well as material- and cost-inefficient. Rational catalyst design is therefore highly desired, but it requires an understanding of the molecular-scale phenomena that govern catalytic functionality. ${ }^{1}$ Such insight into molecular phenomena in complex catalytic systems is scarce and challenging to obtain, owing to the demanding technical requirements associated with imaging chemical interactions and conversion with sufficient selectivity to distinguish different reactants and products, and with high sensitivity to obtain the required spatial resolution. As a result, fundamental aspects-crucial for the development of improved catalysts - remain yet to be fully resolved: (i) Which are the initial chemical transformation steps that occur during and shortly after the adsorption of the reactants and that govern the overall reaction pathways? (ii) Where are the active sites located, and what is the spatio(temporal) distribution of reagents and (intermediate) products on a single catalyst particle?

In this work, we address these questions for an important model system in renewable catalysis: alkene activation and glycol etherification catalyzed by large crystal H-beta zeolites.
Our aim is to elucidate the initial interaction steps of the pure reagents, 1-octene and 1,2-propylene glycol, with the zeolite as well as the onset of the etherification reaction, both during the heating stage, with the use of advanced spectromicroscopies. The reaction has been shown to occur at moderate pressure and an optimum temperature of $140{ }^{\circ} \mathrm{C}$ with high conversion and selectivity, ${ }^{2,3}$ but the activation mechanism has yet to be clarified. Spectroscopic evidence of product ether on the zeolite catalyst could provide insight into the "true", in situ conversion, while identifying the reactive regions at the single particle level would offer crucial knowledge about the spatial distribution of active sites on the catalyst.

This etherification reaction serves as a showcase for numerous processes in which unsaturated hydrocarbons play a major role, such as alkylation or oligomerization. Zeolites, crystalline microporous $\mathrm{Si} / \mathrm{Al}$-oxides, constitute an important class of catalysts for the efficient production of valuable chemicals, for example, transportation fuels and bulk and fine chemicals. ${ }^{4,5}$ The chosen system is prototypical for liquid-phase reactions in which reagents of widely varying polarity need to interact with the heterogeneous catalyst and each other.

In general, conventional bulk catalyst characterization techniques or, more recently, in situ microscopy studies have

Received: September 27, 2011

Published: November 28, 2011 
aimed at spatiotemporal screening of zeolite performance at various temperatures and pressures. These efforts have contributed to our understanding of predominantly the product side of catalytic processes. ${ }^{6-8}$ However, the in situ techniques employed so far have inherent limitations, such as lack of chemical information and limited spatial resolution when working with infrared light or NMR, the need for fluorescent species or labels for fluorescence microscopy, or the typically very low Raman scattering intensities in conventional Raman microscopy.

Here, we map the model catalytic process under study with unprecedented chemical and spatial resolution with coherent anti-Stokes Raman scattering (CARS) spectromicroscopy, a nonlinear, multiphoton analogue of normal Raman scattering. CARS offers quantitative, label-free chemical contrast based on characteristic vibrational resonances of molecules. ${ }^{9-11}$ The exceptional CARS signal strength due to the stimulated excitation of coherent oscillator motions allows for fast chemical mapping of entire zeolite crystals loaded with the reagents, as sketched in Figure 1. Complementary information

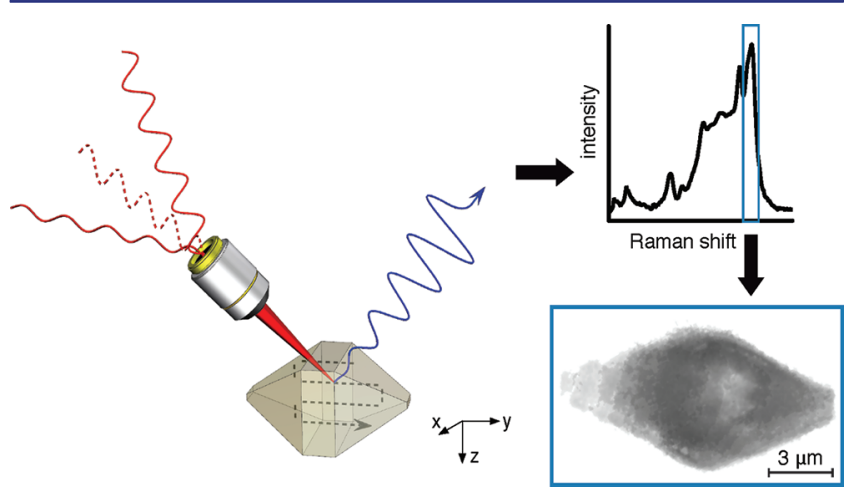

Figure 1. Focusing a multiphoton excitation beam onto a zeolite crystal loaded with reagents (left) locally generates a CARS or $2 \mathrm{PF}$ signal in the focal volume (upper right). Point-by-point mapping of this signal allows the reconstruction of $3 \mathrm{D}$ chemical CARS or $2 \mathrm{PF}$ maps of the catalyst particle (lower right) that quantitatively depict the spatial distribution of different species (voxel size approximately $0.5 \times$ $\left.0.5 \times 2 \mu \mathrm{m}^{3}\right)$.

on fluorescent reaction products is obtained with two-photon fluorescence (2PF) spectromicroscopy. ${ }^{12}$ In addition to submicrometer spatial resolution in the focus plane, both multiphoton excitation techniques offer intrinsic axial resolution of $<2 \mu \mathrm{m}$, allowing true, pinhole-free z-sectioning. Thus, the combination of these techniques provides an ideal platform for in situ, label-free three-dimensional (3D) investigation of chemical conversion processes in heterogeneous catalysis, such as the zeolite-mediated alkene activation and glycol etherification.

\section{EXPERIMENTAL SECTION}

H-Beta Samples. Large (ca. $9 \mu \mathrm{m}$ ) H-beta crystals with a bulk Si/ $\mathrm{Al}$ ratio of 30 (and $2 \mu \mathrm{m}$ large crystals with a bulk $\mathrm{Si} / \mathrm{Al}$ ratio of 20 ; for results see the Supporting Information) were synthesized according to the method of Sano and co-workers ${ }^{13}$ and loaded with 1,2-propylene glycol, 1-octene, or a mixture of the two reagents under similar conditions (molar ratio, Ar pressure, reaction temperature), as described previously. ${ }^{2,3}$ The catalyst samples were taken out upon reaching prespecified temperatures unless noted otherwise, washed with acetone and ethanol, dried under $\mathrm{N}_{2}$ flow, stored in plastic sample containers, and used for spectroscopic analysis.
Multiplex CARS Spectroscopy. Our multiplex CARS setup consists of two tunable mode-locked Ti:sapphire lasers (Tsunami, Spectra Physics) with a repetition rate of $80 \mathrm{MHz}$, pumped by a 532 $\mathrm{nm} \mathrm{Nd}: \mathrm{YVO}_{4}$ laser (Millenium, Spectra Physics). The pump laser was centered at $707 \mathrm{~nm}$ and generated pulses of $10 \mathrm{ps}$. The Stokes laser produced $80 \mathrm{fs}$ pulses and was centered at $910 \mathrm{~nm}$ to probe molecular vibrations with Raman shifts between -2900 and $-3300 \mathrm{~cm}^{-1}$ (i.e., the $\mathrm{CH}$ stretch region) and at $790 \mathrm{~nm}$ to probe the -1300 to -1500 $\mathrm{cm}^{-1} \mathrm{CC}$-stretch region. The total laser power for the experiments varied between 15 and $150 \mathrm{~mW}$ at $0.5 \mathrm{~s}$ integration time. The two beams were adjusted to be collinear and synchronized (Lok-to-Clock, Spectra Physics) to establish the spatial and temporal overlap necessary for the generation of CARS. An additional, home-built feedback system stabilized the long-term time jitter between the laser pulses below 1 ps.

After the raw CARS spectra were divided by a nonresonant reference spectrum (glass coverslip), the resulting normalized data were analyzed by applying the maximum entropy method (MEM) ${ }^{14}$ This mathematical analysis procedure allows us to retrieve the imaginary part of the resonant contribution to the third-order nonlinear susceptibility of the sample, $\operatorname{Im}\left\{\chi^{(3)}\right\}$, which is comparable to the normal Raman response of the system and to which we refer as "CARS response" in this article. To account for small variations in relative band intensities between different crystals, the presented spectra are average spectra of five single-particle CARS responses unless otherwise noted.

Single-Vibration CARS/2PF Imaging. Single-vibration CARS and $2 \mathrm{PF}$ are implemented on a microscopy setup consisting of a picosecond pulsed laser system generating two synchronized beams collinearly aligned into an inverted microscope (Eclipse TE-200, Nikon, Japan) via a beam scanning unit (C1, Nikon). A fraction of the fundamental output of a Nd:Van laser (Picotrain, HighQ Lasers $\mathrm{GmbH}$, Austria) at $1064 \mathrm{~nm}$ is directly coupled into the microscope serving as the Stokes beam in the CARS process, while its frequencydoubled output $(532 \mathrm{~nm})$ is used to synchronously pump an optical parameter oscillator (Emerald OPO, APE GmbH, Germany).

For CARS experiments, the laser beams were focused on the sample by an oil immersion objective (Plan Fluor 40×, NA 1.30, Nikon), resulting in a power of $\sim 10 \mathrm{~mW}$ for each of the beams at the sample position. The signal was collected by an aspherical lens (NA 0.68) in the forward direction and detected by a single-photon-counting photomultiplier tube (PMC-100, Hamamatsu) connected to a timecorrelated single-photon-counting unit (SPCM-830, Becker and Hickl). Bandpass filters in front of the detector suppressed the radiation at the laser wavelengths and transmitted the CARS signal generated in a spectral region between 625 and $675 \mathrm{~nm}$.

For $2 \mathrm{PF}$, the same experimental configuration was employed, although the signal was collected in the epi-direction. Here the signal was obtained by excitation with a single beam from the OPO output (incidence wavelength, $E_{\text {inc }}=811 \mathrm{~nm}$; resulting two-photon excitation wavelength, $E_{\text {exc }}=405 \mathrm{~nm}$ ). The signal, $E_{\text {fluor }}$, was detected in the spectral window between 520 and $550 \mathrm{~nm}(\mathrm{C}=\mathrm{C}$ stretch at -1638 $\left.\mathrm{cm}^{-1}\right)$.

Three-dimensional imaging was achieved by scanning a sequence of horizontal planes at different vertical positions by translating the objective with a motorized stage. Samples were first imaged in brightfield, and the area of interest, typically covering $12 \times 12 \mu \mathrm{m}$, was then imaged at different vertical positions with $1 \mu \mathrm{m}$ spacing by CARS and $2 \mathrm{PF}$ microscopy. The total acquisition time for each layer was $20 \mathrm{~s}$ for both techniques.

\section{RESULTS AND DISCUSSION}

Chemical Characterization. Crystalline, micrometer-sized $\mathrm{H}$-beta zeolite particles were mixed with either liquid 1-octene, 1,2-propylene glycol, or both reagents and reacted in a batch reactor. The batches were removed from the reactor upon reaching prespecified temperatures and subsequently analyzed with CARS/2PF on the single-particle level. Figure 2 shows the CARS responses in the $\mathrm{CH}$ stretch region (centered around 

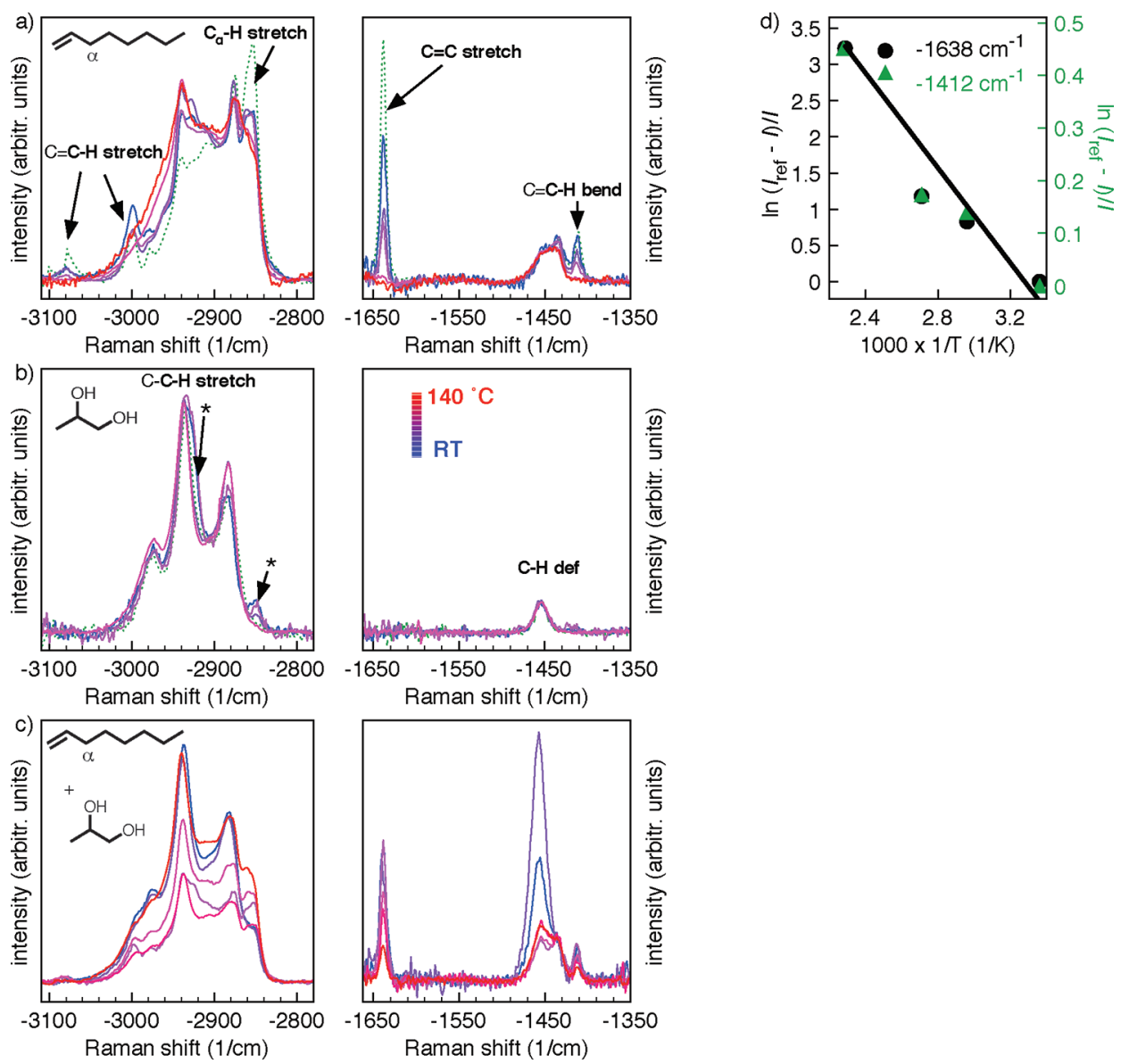

Figure 2. CARS responses from the crystal center of (a) 1-octene, (b) 1,2-propylene glycol, and (c) 1-octene/1,2-propylene glycol mixture adsorbed at H-beta for increasing reaction temperatures (blue to red). Spectra of the pure liquids are shown as reference (dotted green); asterisks denote solvent bands; voxel size approximately $0.5 \times 0.5 \times 2 \mu \mathrm{m}^{3}$. Spectra in the $\mathrm{C}=\mathrm{C}$ region (right) are normalized to the band at ca. $-1445 \mathrm{~cm}^{-1} .(\mathrm{d})$ Van't Hoff plot of logarithm of normalized band intensity ratios $\left(I_{\text {ref }}-I\right) / I$ at $1638 \mathrm{~cm}^{-1}$ (black dots) and at $1412 \mathrm{~cm}^{-1}$ (green triangles) versus $1 / T$ from data in panel a; linear best fit (solid black line).

$2950 \mathrm{~cm}^{-1}$ ) and in the CC stretch region (centered around $1500 \mathrm{~cm}^{-1}$ ) recorded in the center of single zeolite crystals that were removed from the reactor at temperatures between room temperature (blue) and $140{ }^{\circ} \mathrm{C}$ (red). The CARS spectra of the pure liquid reagents are shown for comparison (dotted green). For 1-octene, we observe a convoluted yet characteristic $\mathrm{CH}$ stretch signal between -2840 and $-3100 \mathrm{~cm}^{-1}$ and welldefined bands in the $\mathrm{CC}$ stretch/ $\mathrm{CH}$ bend region at ca. -1450 and $-1650 \mathrm{~cm}^{-1}$ (Figure 2a). 1,2-Propylene glycol exhibits three prominent modes in the $\mathrm{CH}$ stretch region between -2840 and $-3000 \mathrm{~cm}^{-1}$ and one weaker feature in the CC stretch/ $\mathrm{CH}$ bend region at ca. $-1450 \mathrm{~cm}^{-1}$ (Figure $2 \mathrm{~b}$ ). Bands marked with an asterisk originate from ethanol/acetone solvent used in the washing process prior to spectromicroscopy measurements. The spectra of the mixtures (Figure 2c) show a convolution of the single species' signatures, albeit of different relative contributions for each measured temperature. Owing to the high signal-to-noise ratio of the CARS spectra, the resonances can be readily assigned with reference to the literature (see Table 1 in the Supporting Information). The results of similar CARS experiments carried out with individual $2 \mu \mathrm{m}$ sized $\mathrm{H}$-beta particles approaching industrially relevant catalyst dimensions can be found in the Supporting Information.

1-Octene Chemisorption and Formation of Carbocations. The series of 1-octene samples (Figure 2a) shows a strong signal dependence on the reaction temperature. As denoted by arrows, all modes associated with the $\mathrm{C}=\mathrm{C}$ double bond decrease in relative band intensity with increasing temperature. This is indicative of chemisorption of 1-octene at the Brønsted acid sites (BAS) of H-beta (Figure 3, reaction 1a) whereby the zeolite proton is transferred to the alkene, resulting in the formation of a carbocation. Upon the system reaching $120{ }^{\circ} \mathrm{C}$, the $\mathrm{C}=\mathrm{C}$ signatures of the alkene have completely vanished, and the spectral signature closely resembles the Raman spectrum of saturated octane. No spectral features indicating covalently bound alkoxy species of 1-octene (e.g., $\mathrm{Si}-\mathrm{O}-\mathrm{C}$ stretch bands at $\left.1000-1200 \mathrm{~cm}^{-1}\right)^{15}$ are observed. We thus assign the CARS response to octyl carbenium ions strongly stabilized by the zeolite framework. Such ion pair formation has indeed been previously concluded from NMR data for 1-octene adsorption on H-ZSM-5 zeolite ${ }^{16}$ and calculated ${ }^{17,18}$ to be favorable for long-chain alkenes on acidic zeolites, in contrast to stable alkoxy species found for small olefins.

Alternatively, oligomerization of 1-octene could also result in the disappearance of the $\mathrm{C}=\mathrm{C}$ bond signatures. While commonly observed for shorter chain alkenes $(\mathrm{C} 1-\mathrm{C} 4)$ at room temperature, dimerization or trimerization of longer chain olefins like 1-octene was shown to occur only over prolonged times at elevated reaction temperatures $(4 \mathrm{~h}$ at 125 $\left.{ }^{\circ} \mathrm{C}\right) .{ }^{19}$ In previous studies of the 1,2-ethylene glycol/1-octene 

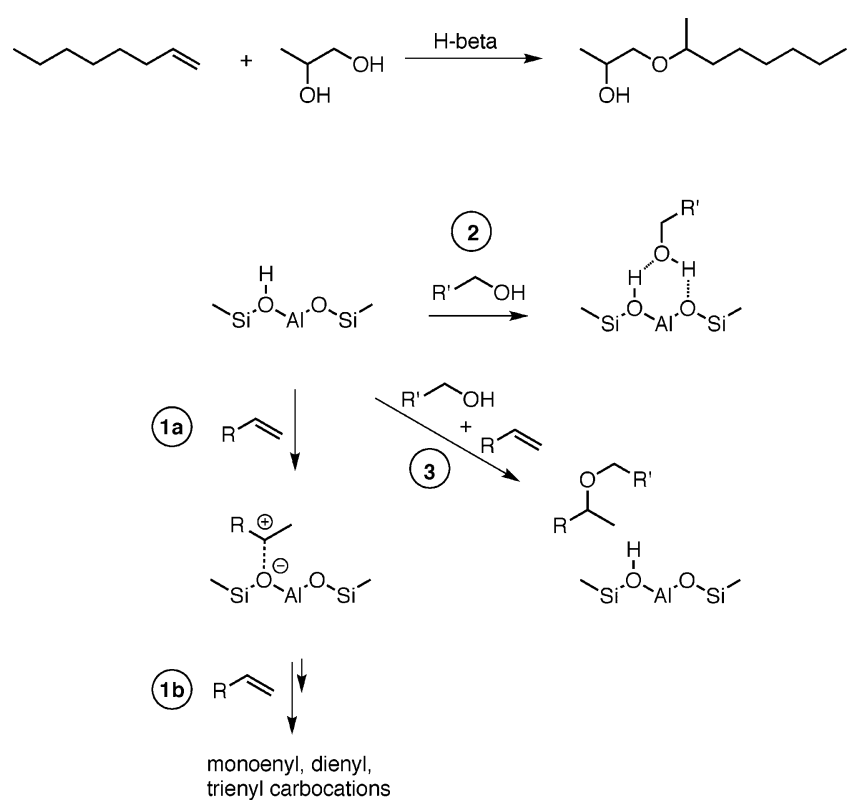

Figure 3. Schematic illustrations of the interaction of the single reagents with the zeolite and of the actual etherification reactions in line with the CARS and 2PF results. Reaction la, alkene activation, formation of octyl carbocation; reaction $1 \mathrm{~b}$, alkene activation, formation of polyenyl cation side products; reaction 2, glycol activation through physisorption; and reaction 3, direct etherification reaction.

etherification, no oligomers were found in the reaction mixture, and alkene oligomerization was implicated as the cause for catalyst deactivation only at longer times and a reaction temperature of $200{ }^{\circ} \mathrm{C} .{ }^{20,21}$ Therefore, we rule out that the disappearance of the double bond signatures during the heating stage up to $140{ }^{\circ} \mathrm{C}$ is caused by oligomerization of 1-octene.

We extract an apparent adsorption enthalpy, $\Delta H_{\mathrm{app}}^{0}$, for 1octene chemisorption from the slope of a Van't Hoff plot of $\ln \left(I_{0}-I\right) / I$ of the double bond marker bands at -1638 (black dots) and $-1412 \mathrm{~cm}^{-1}$ (green triangles) versus $1 / T$ (Figure 2d) of around $19 \pm 4 \mathrm{~kJ} / \mathrm{mol}$ (see Supporting Information for details). This value provides a spatially resolved estimate for 1octene adsorbed in the center of the H-beta crystals. Interestingly, as shown and discussed below, the zeolites do not show a spatially uniform activity: in the shell of the crystal, (side) product formation occurs already at room temperature and then progresses toward the inside with increasing temperature.

While the intense CARS signals show saturated carbocations to be the predominant activation product of 1-octene adsorbed at H-beta, the yellow coloration of the zeolite crystals that intensifies with temperature and correlates with previously observed UV/vis absorption bands at 307, 374, and $460 \mathrm{~nm}^{2}$ suggests the additional formation of polyenyl carbenium ions in the zeolite according to reaction $1 \mathrm{~b}$ in Figure 3. The UV/vis response (not shown) is in very good agreement with monoenyl $(300 \mathrm{~nm})$, dienyl $(360 \mathrm{~nm})$, and trienyl $(460 \mathrm{~nm})$ carbenium ions reported in the literature for various alkene/ zeolite combinations. ${ }^{22,23}$ Note that fluorescence cross sections are considerably (up to 10 orders of magnitude) larger than Raman scattering cross sections. Therefore, the absence of bands characteristic for such conjugated systems in the CARS spectra between -1505 and $-1580 \mathrm{~cm}^{-122,24}$ does not exclude formation of small amounts of such species, below the CARS detection limit of a few millimoles.

1,2-Propylene Glycol Adsorption. The spectral signature of the alcohol (Figure 2b) does not change upon adsorption on the $\mathrm{H}$-beta crystal or with increasing temperature up to $100{ }^{\circ} \mathrm{C}$ . From previous spectroscopic results, it was deduced that alcohol adsorption on acid zeolites occurs through hydrogen bonding between the zeolite proton and the alcohol hydroxyl group, stabilized through interaction with the oxygen of the zeolite framework (Figure 3, reaction 2). ${ }^{25}$ We find that the alcohol vibrational frequencies for liquid and physisorbed 1,2propylene glycol are indistinguishable in the inspected spectral regions, which is in line with recent theoretical results by Nguyen et al. for similar systems. ${ }^{25}$ Apparently, the $\mathrm{CH}$ vibrational modes are largely unaffected upon adsorption of glycol at zeolite acidic sites.

Investigations of the $\mathrm{OH}$ stretch region between -3900 and $-3300 \mathrm{~cm}^{-1}$ could provide more conclusive information about alcohol-zeolite interactions during the heating stage, being sensitive to both the alcohol hydroxyl group and the BAS of the catalyst. However, experiments at these anti-Stokes shifts are not feasible with our current instrumentation.

Reaction Mixture. The liquid-phase, solventless etherification requires two reactants of opposing polarity to react on the catalyst. It is therefore essential to gain information about the activation and distribution of the two components over the catalyst particle. To monitor the actual etherification reaction (Figure 3, reaction 3), we measured zeolite particles reacted at different temperatures up to $140{ }^{\circ} \mathrm{C}$ with both reagents, 1-octene and 1,2-propylene glycol. Similar to the case of pure 1-octene, the crystals loaded with the reagent mixture are of yellow color, albeit less intense. The CARS responses (Figure 2c) were again recorded in the center of the particles.

We obtain a quantitative estimate of the relative contribution of different species to the spectral responses from non-negative least-squares fitting carried out with Wavemetrics Igor Pro 6.21, based on an algorithm by Bro and De Jong. ${ }^{26}$ The spectrum of 1-octene/H-beta heated to $70{ }^{\circ} \mathrm{C}$, to account for both alkene and alkyl cation contributions, and the spectrum of 1,2propylene glycol/H-beta at $100{ }^{\circ} \mathrm{C}$, as presented in Figure 2a,b, respectively, served as reactant references. The product reference was measured on product ether previously synthesized and purified and subsequently adsorbed on H-beta crystals. The reliability of the employed fit procedure was tested extensively, varying the relative contributions of the three components to an overall spectrum and adding various levels ( 0.1 to $0.01 \%)$ of Gaussian noise, showing an error of $\leq 5 \%$.

The spectral analysis clearly indicates ether formation on zeolite particles removed from the reactor immediately upon reaching $140{ }^{\circ} \mathrm{C}\left(t_{0}\right.$, Figure $\left.4 \mathrm{a}\right)$ as well as on samples that were left to further react at $140{ }^{\circ} \mathrm{C}$ for $90 \mathrm{~min}\left(t_{90}\right.$, Figure $\left.4 \mathrm{~b}\right)$, while no ether is detected up to a reaction temperature of $120^{\circ} \mathrm{C}$ on the majority of the probed H-beta particles (data not shown). To the best of our knowledge, this is the first direct spectroscopic evidence of the product ether observed on a zeolite catalyst. While for $t_{0}$ crystals we find a relative ether signal contribution to the overall signal intensity between 5 and $11 \%$ (Figure 4a), this contribution strongly increases after 90 min to values of $50 \pm 24 \%$ (Figure $4 \mathrm{~b}$ ). Large crystal-to-crystal variations were observed in the amount of ether detected. These variations can be traced to variations in initial reactant concentrations: For three out of five particles, glycol conversion is complete (no relative signal contribution of glycol to overall 

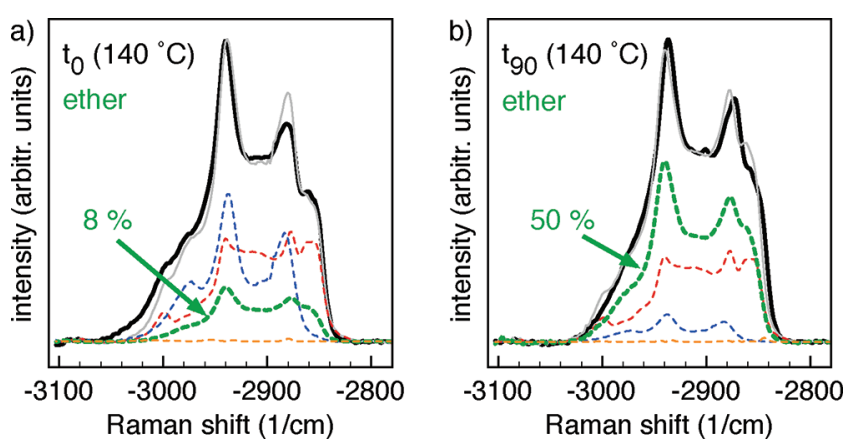

Figure 4. Examples of CARS responses of a mixture of 1-octene and 1,2-propylene glycol adsorbed at $\mathrm{H}$-beta at $140{ }^{\circ} \mathrm{C}$ (solid black) (a) after $0 \mathrm{~min}$ and (b) after $90 \mathrm{~min}$ and non-negative least-squares fit (gray; relative remainder (\%), dotted orange) of single reagents' spectra (1-octene/octyl cation, dotted red; 1,2-propylene glycol, dotted blue) and of product ether spectrum (thick dotted green). The relative contribution of ether to the overall signal increases with time from $8 \pm 3 \%\left(t_{0}\right)$ to $50 \pm 24 \%\left(t_{90}\right)$. See text for details.

signal) at $t_{90}$, while in one case only a few percent of 1-octene remains on the crystal. No trend is observed with respect to the relative amounts of alkene/glycol with varying temperature or position on the particle. The factor $\sim 5$ discrepancy between off-line ether yields of around $10 \%$ for $t_{90}$ samples as previously determined with gas chromatography ${ }^{2}$ with the on-particle relative ether amount found in this study suggests that the overall product yield is limited by incomplete product desorption during the retrieval process.

Our results demonstrate the correlation between ether formation and alkene activation: Similar to the case of pure 1-octene adsorbed at $\mathrm{H}$-beta, the alkene $\mathrm{C}=\mathrm{C}$ marker bond intensities decrease with increasing temperature also for the reactant mixture. However, as evident from the remaining $\mathrm{C}=$ $\mathrm{C}$ bond band intensities at $140{ }^{\circ} \mathrm{C}$, the alkene in the reaction mixture is not activated completely in the studied temperature range (Figure 2c, CC region normalized to the band at ca. $\left.-1445 \mathrm{~cm}^{-1}\right)$, in contrast to our observations for 1-octene/Hbeta. This is consistent with the notion that the alkene activation mechanism is not altered due to the presence of 1,2propylene glycol. Nonetheless, the formation of octyl cations is suppressed, indicating that the accessibility of the BAS is limited in the presence of 1,2-propylene glycol. This is not surprising since the adsorption of 1,2-propylene glycol on $\mathrm{H}$ beta is favored over the adsorption of 1-octene due to the hydrophilic nature of the zeolite. In addition, proton solvation by the excess alcohol may further impair the formation of carbocations from the alkene.

We conclude that the activation of 1-octene is the first reaction step toward etherification of 1,2-propylene glycol and occurs already below $100{ }^{\circ} \mathrm{C}$. The preferred adsorption of the glycol over the alkene appears to be rate-controlling, with diffusion of alkene and alcohol to and away from, respectively, reactive BAS requiring elevated temperatures of $\geq 140{ }^{\circ} \mathrm{C}$ and prolonged reaction times.

3D Reactivity Maps. To identify the most reactive regions of the H-beta zeolites for alkene activation, i.e., the crucial step toward glycol etherification, we performed 3D CARS and 2PF imaging of $\mathrm{H}$-beta particles loaded with 1-octene. While $\mathrm{C}=\mathrm{C}$ stretch-band maps identify exclusively the unreacted alkene with an intact double bond on the crystal, the 2PF maps depict the distribution of the fluorescent polyenyl cations that form as a side product in minor quantities during alkene activation. The spatial evolution of the alkene (CARS $\mathrm{C}=\mathrm{C}$ band at -1638 $\mathrm{cm}^{-1}$, red) and polyenyl cation (2PF, green) densities with temperature recorded in the center plane of a particle is shown in Figure 5. The particle dimensions observed in CARS and
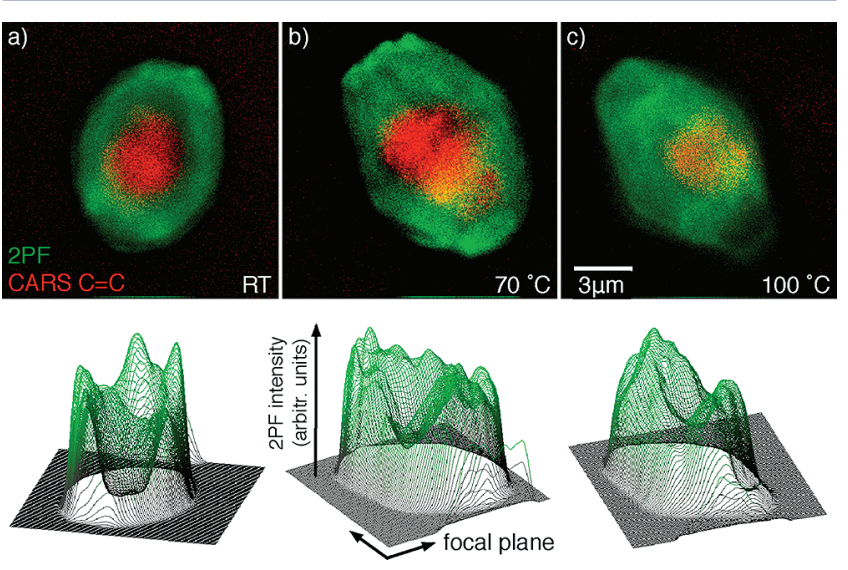

Figure 5. Overlays of CARS and $2 \mathrm{PF}$ chemical maps of 1-octene adsorbed at $\mathrm{H}$-beta at (a) room temperature, (b) $70^{\circ} \mathrm{C}$, and (c) 100 ${ }^{\circ} \mathrm{C}$. Red, single-line CARS map of $\mathrm{C}=\mathrm{C}$ stretch band intensity at $-1638 \mathrm{~cm}^{-1}$; green, $2 \mathrm{PF}$ map $\left(E_{\text {inc }}=811 \mathrm{~nm}, E_{\text {fluor }}=520-550 \mathrm{~nm}\right)$. The corresponding $2 \mathrm{PF}$ intensity profiles are shown below the maps. Images were recorded in the center plane of the crystal.

2PF maps excellently match the catalyst size as inferred from white light microscopy. At room temperature, the fluorescent side products are mainly found in the outer shell of the model crystals (compare intensity profiles below maps in Figure 5), and the alkene remains unreacted in the center of the crystals. With increasing temperature, activation of 1-octene and the formation of polyenyl cations are also observed in the inner region of the zeolite. Still, the fluorescence remains more pronounced in the shell. Reconstructed 3D videos of the fluorescent side product distribution are available in the Supporting Information.

The spatially inhomogeneous reactivity throughout a single zeolite crystal is not likely caused by hindered reagent diffusion during the loading of 1-octene, as already at room temperature, the entire body of the zeolite particles is filled with reagent (red regions from $\mathrm{CARS} \mathrm{C}=\mathrm{C}$ signatures in Figure 5). However, our observations can be ascribed to aluminum zoning, i.e., differences in the $\mathrm{Si} / \mathrm{Al}$ ratio between various regions of the zeolite, a phenomenon often observed for zeolites. ${ }^{27,28}$ The etherification activity of H-beta is based on the presence of BAS in the zeolite. The acid strength of a zeolite is determined by its $\mathrm{Si} / \mathrm{Al}$ ratio, i.e., the number of acidic sites, and by the BAS distribution in the crystal. By comparing XPS data for surface $\mathrm{Al}$ content and ICP data for bulk composition, we previously found that H-beta crystals, such as the ones employed in this study, show a higher $\mathrm{Al}$ concentration on the surface than in the bulk. $^{2}$ This effect was observed to become more pronounced with increasing particle size. Furthermore, the higher $\mathrm{Al}$ content in the outer rim of the large $\mathrm{H}$-beta crystals entails the presence of more extraframework $\mathrm{Al}$ Lewis acid sites that are known to increase the reactivity of neighboring $\mathrm{BAS}^{29,30}$ The locally higher $\mathrm{Si} / \mathrm{Al}$ ratio in the center of the particle results in BAS which are less active than the BAS in the rim because of the absence of extraframework Al. This is in line with the observed polyenyl cation formation over $1-3 \mu \mathrm{m}$ in the rim of the zeolite already at room temperature, while in the inner region elevated 
temperatures from $100{ }^{\circ} \mathrm{C}$ are required for the same process to occur. Indeed, several CARS spectra recorded of H-beta particles loaded with both reagents suggest the presence of a few percent of ether in the shell $(1-2.5 \mu \mathrm{m}$ width) in samples heated to $100{ }^{\circ} \mathrm{C}$ (data not shown), where definitely no product is found in the center of the particles.

\section{CONCLUSION}

We have gained detailed understanding of 1-octene activation and the onset of 1,2-propylene glycol etherification with 1octene on single H-beta zeolite crystals with unprecedented chemical specificity and spatial resolution using CARS and $2 \mathrm{PF}$ microscopies. The activation step is the chemisorption of the alkene throughout the entire zeolite particle and the formation of strongly stabilized octyl carbocations in the prereaction temperature range $\left(<140{ }^{\circ} \mathrm{C}\right)$. During alkene activation, trace amounts of polyenyl carbenium ions are formed which are responsible for the yellow coloration of the crystals and their strong $2 \mathrm{PF}$ emission. As evident from 3D CARS and 2PF maps, with increasing temperature the alkene activation and polyenyl cation formation progress from the outer rim to the inner regions of the of the $\mathrm{H}$-beta particles, which is explained in terms of $\mathrm{Al}$ zoning in the crystals. The presence of glycol clearly suppresses 1-octene activation. Thus, as a prerequisite for glycol etherification, elevated temperatures of $140{ }^{\circ} \mathrm{C}$ and prolonged reaction times are required to facilitate reagent mobility on the crystal and allow for quantitative alkene activation and glycol conversion. We provide first direct spectroscopic evidence for the product ether on the zeolite crystals and find a relative onparticle ether concentration of $50 \pm 24 \%$ after $90 \mathrm{~min}$ at 140 ${ }^{\circ} \mathrm{C}$, ca. 5 times higher than off-line conversion estimates. This discrepancy is attributed to incomplete ether desorption during the product recovery process.

In addition to elucidating details of the local chemical conversion for this important model reaction, our findings demonstrate that nonlinear spectromicroscopies are a novel valuable addition to the methodological pool employed in heterogeneous catalysis.

\section{ASSOCIATED CONTENT}

\section{S Supporting Information}

Synthesis of H-beta crystals; table of Raman bands and assignment; derivation of the apparent adsorption enthalpy of 1-octene on H-beta, $\Delta H_{\text {app }}^{0}$; reconstructed $3 \mathrm{D}$ videos of the fluorescent side product distribution; CARS and UV/vis results of $2 \mu \mathrm{m}$ large H-beta crystals. This material is free of charge via the Internet at http://pubs.acs.org.

\section{AUTHOR INFORMATION}

\section{Corresponding Author}

domke@amolf.nl

\section{ACKNOWLEDGMENTS}

The authors thank Davide Mores for supplying the UV/vis data. K.F.D. thanks the Alexander von Humboldt Foundation (Germany) for a Feodor Lynen Fellowship. Imaging CARS microscopy was made available by generous support from the Swedish Research Council. This work is part of the research program of the Stichting FOM with financial support from NWO.

\section{REFERENCES}

(1) Christensen, C. H.; Nørskov, J. K. J. Chem. Phys. 2008, 128, 182503.

(2) Parvulescu, A. N.; Mores, D.; Stavitski, E.; Teodorescu, C. M.; Bruijnincx, P. C. A.; Klein Gebbink, R. J. M.; Weckhuysen, B. M. J. Am. Chem. Soc. 2010, 132, 10429-10439.

(3) Ruppert, A. M.; Parvulescu, A. N.; Arias, M.; Hausoul, P. J. C.; Bruijnincx, P. C. A.; Klein Gebbink, R. J. M.; Weckhuysen, B. M. J. Catal. 2009, 268, 251-259.

(4) Vispute, T. P.; Zhang, H.; Sanna, A.; Xiao, R.; Huber, G. W. Science 2010, 330, 1222-1227.

(5) Busca, G. Chem. Rev. 2007, 107, 5366-5410.

(6) Cremer, G. D.; Sels, B. F.; Vos, D. E. D.; Hofkens, J.; Roeffaers, M. B. J. Chem. Soc. Rev. 2010, 39, 4703-4717.

(7) Ivanova, I. I.; Kolyagin, Y. G. Chem. Soc. Rev. 2010, 39, 50185050 .

(8) Weckhuysen, B. M. Angew. Chem., Int. Ed. 2009, 48, 4910-4943.

(9) Day, J. P.; Domke, K. F.; Rago, G.; Kano, H.; Hamaguchi, H.; Vartiainen, E. M.; Bonn, M. J. Phys. Chem. B 2011, 115, 7713-7725.

(10) Kox, M. H. F.; Domke, K. F.; Day, J. P. R.; Rago, G.; Stavitski, E.; Bonn, M.; Weckhuysen, B. M. Angew. Chem., Int. Ed. 2009, 48, $8990-8994$

(11) Müller, M.; Zumbusch, A. ChemPhysChem 2007, 8, 2156-2170.

(12) Carriles, R.; Schafer, D. N.; Sheetz, K. E.; Field, J. J.; Cisek, R.; Barzda, V.; Sylvester, A. W.; Squier, J. A. Rev. Sci. Instrum. 2009, 80, 081101.

(13) Lu, B.-W.; Jon, H.; Kanai, T.; Oumi, Y.; Itabashi, K.; Sano, T. J. Mater. Sci. 2006, 41, 1861-1864.

(14) Vartiainen, E. M.; Rinia, H. A.; Müller, M.; Bonn, M. Opt. Exp. 2006, 14, 3622-3630.

(15) Saito, N.; Lee, S.-H.; Maeda, N.; Ohta, R.; Sugimur, H.; Takai, O. J. Vac. Sci. Technol. A 2004, 22, 1425-1427.

(16) Stepanov, A. G.; Luzgin, M. V.; Romannikov, V. N.; Zamaraev, K. I. Catal. Lett. 1994, 24, 271-284.

(17) Fang, H.; Zheng, A.; Li, S.; Xu, J.; Chen, L.; Deng, F. J. Phys. Chem. C 2010, 114, 10254-10264.

(18) Boronat, M.; Viruela, P.; Corma, A. J. Am. Chem. Soc. 2004, 126, $3300-3309$

(19) Alonso, D. M.; Bond, J. Q.; Serrano-Ruiz, J. C.; Dumesic, J. A. Green Chem. 2010, 12, 992.

(20) Ruppert, A. M.; Parvulescu, A. N.; Arias, M.; Hausoul, P. J. C.; Bruijnincx, P. C. A.; Klein Gebbink, R. J. M.; Weckhuysen, B. M. J. Catal. 2009, 268, 251-259.

(21) de Klerk, A.; Nel, R. J. J. Ind. Eng. Chem. Res. 2007, 46, 70667072.

(22) Kiricsi, I.; Förster, H.; Tasi, G.; Nagy, J. B. Chem. Rev. 1999, 99, 2085-2114.

(23) Cozens, F. L.; Bogdanova, R.; Regimbald, M.; Garcia, H.; Marti, V.; Scaiano, J. C. J. Phys. Chem. B 1997, 101, 6921-6928.

(24) Paze, C.; Sazak, B.; Zecchina, A.; Dwyer, J. J. Phys. Chem. B 1999, 103, 9978-9986.

(25) Nguyen, C. M.; Reyniers, M.-F.; Marin, G. B. Phys. Chem. Chem. Phys. 2010, 12, 9481-9493.

(26) Bro, R.; De Jong, S. J. Chemometrics 1997, 11, 393-401.

(27) van Broekhoven, J. A.; Danilina, N. In Zeolites and catalysis: Synthesis, reactions and applications; Cejka, J., Corma, A., Zones, S., Eds.; Wiley: Weinheim, 2010; Chapter 10.

(28) Danilina, N.; Krumeich, F.; Castelanelli, S. A.; van Bokhoven, J. A. J. Phys. Chem. C 2010, 114, 6640-6645.

(29) Williams, B.; Babitz, S.; Miller, J.; Snurr, R.; Kung, H. Appl. Catal. 1999, 177, 131-175.

(30) Guisnet, M.; Ayrault, P.; Coutanceau, C.; Alvarez, M. F.; Datka, J. J. Chem. Soc., Faraday Trans. 1997, 93, 1661-1665. 